\title{
Exploring Herbicidal Potential of Aqueous Extracts of Some Herbaceous Plants Against Parthenium Weed ${ }^{1}$
}

\author{
Explorando Herbicida Potencial de Aquosas Extratos de Algumas Plantas Herbáceas contra \\ Parthenium Weed
}

SAFDAR, M.E. ${ }^{2}$, TANVEER, A. ${ }^{3}$, KHALIQ, A. ${ }^{3}$, ALI, H.H. ${ }^{3}$, and BURGOS, N.R. ${ }^{4}$

\begin{abstract}
To assess the phytotoxic potential of Achyranthes aspera, Alternanthera philoxeroides, Datura metel and Rumex dentatus against Parthenium hysterophorus, 5\% (w/v on dry weight basis) aqueous extracts from root, stem, leaf, flower and whole plant were tested through a Petri plate-based germination and pot-cultured seedling bioassays. Achyranthes aspera and A. philoxeroides inhibited parthenium weed germination more than extracts from other species. Whole plant, leaf and fruit extracts of $A$. aspera reduced the germination percentage $(5 \%)$; leaf extract from $A$. philoxeroides caused lower germination index (0.4), higher mean germination time $(14 \mathrm{~d})$ and longer time to $50 \%$ germination $(13.5 \mathrm{~d})$ of parthenium weed. In the foliar spray bioassay, A. aspera reduced parthenium weed shoot growth more than the other species whereas $R$. dentatus caused more reduction in root growth. Whole plant extract from $A$. aspera caused maximum reduction in parthenium weed seedling vigor index $(98 \%)$ and seedling biomass (96\%). The aqueous extracts of $A$. aspera and A. philoxeroides contained higher concentrations of phenolics viz. gallic $\left(16.9 \mathrm{mg} \mathrm{L}^{-1}\right)$, caffeic $\left(7.4 \mathrm{mg} \mathrm{L}^{-1}\right)$, chromatotropic (63.8 $\left.\mathrm{mg} \mathrm{L}^{-1}\right)$, $p$-coumaric $\left(10.5 \mathrm{mg} \mathrm{L}^{-1}\right), m$-coumaric $\left(3.1 \mathrm{mg} \mathrm{L}^{-1}\right)$, syringic $\left(9.21 \mathrm{mg} \mathrm{L}^{-1}\right)$ and 4-hydroxy-3-methoxy benzoic (118.6 $\left.\mathrm{mg} \mathrm{L}^{-1}\right)$ acids compared with extracts of the other two species tested.
\end{abstract}

Keywords: allelopathy, bio-herbicides, parthenium weed, seed germination, seedling growth, weed management.

\begin{abstract}
RESUMO - Para avaliar o potencial fitotóxico de Achyranthes aspera, Alternanthera philoxeroides, Datura metel e Rumex dentatus sobre Parthenium hysterophorus, 5\% $(w / v$ em base seca) de extratos aquosos de raiz, caule, folha, flor e toda a planta foram testados através de germinação em placa de Petri e de bioensaios de sementes cultivadas em potes. A inibição da germinação de plantas de Parthenium por Achyranthes aspera e A. philoxeroides foi maior que a de extratos de outras espécies. Extratos de plantas e folhas e frutos inteiros de A. aspera reduziram a porcentagem de germinação (5\%); o extrato de folha de $\boldsymbol{A}$. philoxeroides provocou menor índice de germinação $(0,4)$, maior tempo médio de germinação $(14$ d) e germinação por mais tempo a 50\% (13,5 d) de Parthenium. No bioensaio de pulverização foliar, A. aspera reduziu mais o crescimento de rebentos de Parthenium do que as outras espécies, enquanto $\boldsymbol{R}$. dentatus causou maior redução no crescimento radicular. O extrato de planta inteira de $\boldsymbol{A}$. aspera causou redução máxima do índice de vigor de plântulas (98\%) e da biomassa das plântulas (96\%) de Parthenium. Os extratos aquosos de $\boldsymbol{A}$. aspera $e \boldsymbol{A}$. philoxeroides continham as concentrações mais elevadas de compostos fenólicos, ou seja, ácidos gálico (16,9 $\left.\mathrm{mg} \mathrm{L}^{-1}\right)$, cafeico $\left(7,4 \mathrm{mg} \mathrm{L} \mathrm{L}^{-1}\right)$, cromotrópico $\left(63,8 \mathrm{mg} \mathrm{L}^{-1}\right)$, p-cumárico (10,5 $\left.\mathrm{mg} \mathrm{L}^{-1}\right)$, m-cumárico (3,1 $\left.\mathrm{mg} \mathrm{L}^{-1}\right)$, siríngico $\left(9,21 \mathrm{mg} \mathrm{L}^{-1}\right)$ e benzoico 4-hidroxi-3-metoxi $\left(118,6 \mathrm{mg} \mathrm{L}^{-1}\right)$, em comparação com os extratos das outras duas espécies testadas.
\end{abstract}

Palavras-chave: alelopatia, bio-herbicidas, plantas de Parthenium, germinação das sementes, crescimento de plântulas, manejo de plantas daninhas.

Recebido para publicação em 11.7.2015 e aprovado em 12.10.2015.

Department of Agronomy, University College of Agriculture, University of Sargodha, Sargodha, Pakistan, <ehsan_safdar2002@yahoo.com>; ${ }^{3}$ Department of Agronomy, University of Agriculture, Faisalabad, Pakistan; ${ }^{4}$ Department of Crop, Soil and Environmental Sciences, University of Arkansas Fayetteville AR, USA.

Planta Daninha, Viçosa-MG, v. 34, n. 1, p. 109-116, 2016 


\section{INTRODUCTION}

Parthenium hysterophorus, a member of the Asteraceae family, is commonly known as carrotgrass, whitehead weed, congress grass, or parthenium weed. This weed has invaded about 30 countries in Africa, Australia and Asia, including Pakistan (Chippendale \& Panetta, 1994; Shabbir \& Bajwa, 2007; Nigatu et al., 2010). Parthenium is an obnoxious weed rapidly spreading across the non-cropped areas of the Khyber Pakhtunkhwa province and elsewhere in Pakistan; where it has caused serious damage to pastures, crops and biodiversity (Khan et al., 2012; Masum et al., 2013). Yield losses due to the presence of this weed in various crops range from 33 to $90 \%$ (Tamado et al., 2002; Vivek et al., 2008; Khan et al., 2013). In addition, it also causes allergy, dermatitis and asthma in human and cattle due to its poisonous nature (Suresh et al., 1994; Evans, 1997; Khan et al., 2013).

It is an alien invasive weed spreading throughout Pakistan in both irrigated and rainfed areas (Shabbir \& Bajwa, 2007; Khan et al., 2012). Its presence as an aggressive colonizer in both non-cropped and cropped situations has been well documented (Javaid \& Anjum, 2005; Riaz \& Javaid, 2010; Khan et al., 2014). The widespread invasion of parthenium weed in natural grasslands, field crops, orchards and vegetable fields in the country has prodded weed scientists to devise strategies for its management. Although parthenium weed can be successfully controlled with herbicides (Sharma, 2003; Javaid et al., 2007; Kathiresan, 2008; Khan et al., 2012), chemical control however is not recommended due to its detrimental effects on the environment and human health. In order to minimize the usage of synthetic herbicides, plant extracts alone or in combination with herbicides should be used. These extracts contain potential allelochemicals such as phenolics, terpenoids, alkaloids, coumarins, tannins, flavonoids, steroids and quinines (Xuan et al., 2005), which are capable of selectively and non-selectively influencing plant germination and growth at various concentrations, often at very small concentrations.

Studies have been carried out using various herbaceous plants extracts for their phytotoxic effect against parthenium weed (Anjum et al., 2006; Javaid et al., 2005; Quazi $\&$ Khan, 2010; Shafique et al., 2011). Some of these such as Amaranthus spinosus, Imperata cylindrica, Desmostachya bipinnata, Dichanthium annulatum, Cenchrus pennisetiformis, Physalis minima, Sorghum halepense, Tagetes erectus and Xanthium strumarium were found effective against this weed. Nevertheless, the degree and nature of phytotoxic action of aqueous extract of a plant varies by plant part (Sinha $\&$ Singh, 2004; Swain et al., 2005) and extract concentration (Safdar et al., 2013). Hence, there is a need to test other well-known allelopathic plants to explore their potential phytotoxic activity against parthenium weed. Therefore, some herbaceous plant species were evaluated for their pre and postemergence herbicidal activity against parthenium weed.

\section{MATERIALS AND METHODS}

Germination and seedling growth bioassays were conducted using four herbaceous species viz., Achyranthes aspera, Alternanthera philoxeroides, Datura metel, and Rumex dentatus in petri plates and pots. Studies were carried out at Weed Science Laboratory, Department of Agronomy, University of Agriculture, Faisalabad, Pakistan during 2011-12.

\section{Preparation of aqueous extracts}

Wild growing plants of each species were collected and kept at room temperature for drying and then oven-dried at $70^{\circ} \mathrm{C}$ for $48 \mathrm{~h}$. Intact plants of those species were then separated into root, stem, leaves and fruit. However, of $A$. philoxeroides into root, stem and leaves as fruit were absent in it. Some whole plants of all plant species were also kept intact. These were then cut into small $(2 \mathrm{~cm})$ pieces and separately soaked in distilled water at a ratio of 1: $20 \mathrm{w} / \mathrm{v}$ (herbage:water) for $24 \mathrm{~h}$ at room temperature (Hussain \& Gadoon, 1981). Aqueous extracts (5\% w/v) of different plant parts were obtained by filtering the mixture using an ASTM 40 mesh sieve and then through Sartorious Minisart syringe filters with $0.45 \mu \mathrm{m}$ pore size. 


\section{Determination of biochemical characteristics of aqueous extracts}

The $\mathrm{pH}$ and EC of each extract were determined with a $\mathrm{pH}$ meter (JENWAY $3510 \mathrm{pH}$ Meter) and an EC meter (JENWAY 3510 conductivity Meter), respectively. Total soluble phenolics were determined as described by Randhir \& Shetty (2005) and expressed as gallic acid equivalents (Table 1). For identification and quantification of their suspected phytotoxins, aqueous extracts were chemically analysed using a Shimadzu HPLC system equipped with a UV detector (Model SCL-10A, Tokyo, Japan). The peaks were identified using chemical standards (Aldrich, St Louis, USA). The concentration of each isolated compound (Table 2) was determined using the following equation:

$$
\text { Concentration }(\text { ppm })=\frac{\text { Area of the sample }}{\text { Area of the standard }} \times \text { Concentration of the standard } \times \text { Dilution factor }
$$

Table 1 - Characterization of the chemical properties of various aqueous plant extracts

\begin{tabular}{|c|c|c|c|c|}
\hline Plant & Plant Part & $\mathrm{pH}$ & $\begin{array}{c}\mathrm{EC} \\
\left(\mathrm{dS} \mathrm{m}^{-1}\right)\end{array}$ & $\begin{array}{c}* \text { Concentration of total } \\
\text { phenolics }\left(\mathrm{mg} \mathrm{L}^{-1}\right)\end{array}$ \\
\hline \multirow{5}{*}{ Datura metel L. } & Root & 7.9 & 6.3 & - \\
\hline & Stem & 8.6 & 9.8 & - \\
\hline & Leaf & 8.2 & 10.9 & 5479 \\
\hline & Fruit & 8.8 & 7.0 & - \\
\hline & Whole plant & 8.8 & 8.8 & - \\
\hline \multirow{5}{*}{ Rumex dentatus L. } & Root & 6.8 & 1.0 & - \\
\hline & Stem, & 9.0 & 5.8 & - \\
\hline & Leaf & 8.2 & 8.8 & 4948 \\
\hline & Fruit & 9.1 & 2.1 & - \\
\hline & Whole plant & 9.0 & 3.7 & - \\
\hline \multirow{6}{*}{ Achyranthes aspera L. } & Root & 8.3 & 2.7 & - \\
\hline & Stem & 9.6 & 3.9 & - \\
\hline & Leaf & 9.1 & 5.4 & 3795 \\
\hline & Fruit & 9.4 & 3.8 & - \\
\hline & Whole plant & 9.3 & 4.7 & 5779 \\
\hline & Root & 9.1 & 4.6 & - \\
\hline \multirow{3}{*}{ Alternanthera philoxeroides Gris. } & Stem & 9.2 & 8.3 & - \\
\hline & Leaf & 9.2 & 8.6 & 2618 \\
\hline & Whole plant & 9.4 & 8.2 & - \\
\hline
\end{tabular}

* Concentration of total phenolics were only determined of those aqueous extracts which showed maximum germination inhibition.

Table 2 - Chemical composition of aqueous plant extracts

\begin{tabular}{|r|l|r|r|r|r|}
\hline \multirow{2}{*}{$\begin{array}{c}\text { Sr. } \\
\text { No. }\end{array}$} & \multicolumn{1}{|c|}{ Chenolics } & \multicolumn{4}{c|}{ Concentration $\left(\mathrm{mg} \mathrm{L}^{-1}\right)$} \\
\cline { 3 - 5 } & & D. metel & R. dentatus & A. aspera & A. philoxeroides \\
\hline 1 & Chromatotropic acid & - & - & 63.8 & 13.8 \\
\hline 2 & Gallic acid & - & 17.3 & 16.9 & - \\
\hline 3 & Caffeic acid & - & - & 7.4 & - \\
\hline 4 & Vanillic acid & 26.3 & - & - & - \\
\hline 5 & 4-hydroxy-3-methoxy benzoic acid & 96.7 & - & 24.8 & - \\
\hline 6 & p-Coumaric acid & - & - & 3.1 & 118.6 \\
\hline 7 & m-Coumaric acid & - & - & 9.2 & - \\
\hline 8 & Syringic acid & 51.3 & - & - & - \\
\hline 9 & Ferulic acid & - & - & - & - \\
\hline 10 & Chlorogenic acid & - & - & - & - \\
\hline
\end{tabular}


Biochemical characteristics of aqueous extracts of parthenium plant parts and their rhizospheric soils from various farm locations and their phenolic compositions are presented in Tables 1 and 2.

\section{Germination studies}

To evaluate preemergence herbicidal potential against parthenium, $5 \%$ aqueous extracts of root, stem, leaves, fruit and whole plant of $A$. aspera, D. metel and $R$. dentatus and root, stem, leaves and whole plant of A. philoxeroides were used in the germination bioassay. Twenty seeds of parthenium were placed in $9 \mathrm{~cm}$ dia. Petri plates lined with a double layer of Whatman No. 10 filter paper and moistened with $4 \mathrm{~mL}$ of each extract. A control treatment with $4 \mathrm{~mL}$ of distilled water was included. Each treatment was replicated 4 times and the plates arranged in a completely randomized design with a factorial arrangement of species $X$ extract concentration. Petri plates were kept in an illuminated incubator maintained at $25 \pm 5{ }^{\circ} \mathrm{C}$. Germination was recorded daily for 20 days. Germination count data were used to calculate various seed germination and vigor parameters as detailed below:

Germination percentage (GP) was calculated by the following formula:

$$
\mathrm{GP}=\left[\mathrm{N}_{\mathrm{T}} \times 100\right] / \mathrm{N}
$$

where $\mathrm{N}_{\mathrm{T}}$ : proportion of germinated seeds in each treatment for the final measurement, and $\mathrm{N}$ : Number of seeds used in the bioassay.

The germination index (GI) was calculated by following the formula as described by Scott et al. (1984):

$$
\mathrm{GI}=\mathrm{N}_{1} / \mathrm{D}_{1}+\ldots \ldots \ldots \ldots \ldots \ldots+\mathrm{N}_{\mathrm{L}} / \mathrm{D}_{\mathrm{L}} \text { (eq. } 3 \text { ) }
$$

where $\mathrm{N}_{1}$ : number of seeds germinated in 1 st count, $\mathrm{D}_{1}$ : days to 1 st count, $\mathrm{N}_{\mathrm{L}}$ : number of seeds germinated in last count, and $\mathrm{D}_{\mathrm{L}}$ : days to last count.

Mean germination time (MGT) was calculated by the following equation, as given by Dezfuli et al. (2008):

$$
\mathrm{MGT}=\Sigma \mathrm{Dn} / \Sigma \mathrm{n}
$$

where $\mathrm{n}$ : Number of seeds which were germinated on day D, D: Number of days counted from the beginning of the germination.

Time to $50 \%$ germination $\left(\mathrm{T}_{50}\right)$ was calculated according to the following formula modified by Farooq et al. (2005):

$$
\mathrm{T}_{50}=\mathrm{t}_{\mathrm{i}}+\left\{(\mathrm{N} / 2)-\mathrm{n}_{\mathrm{i}}\right\}\left(\mathrm{t}_{\mathrm{j}}-\mathrm{t}_{\mathrm{i}}\right) / \mathrm{n}_{\mathrm{j}}-\mathrm{n}_{\mathrm{i}} \text { (eq. 5) }
$$

where N: Final number of germination, $n_{i}, n_{j}$ : cumulative number of seeds germinated by adjacent counts at times $t_{i}$ and $t_{j}$ when $\mathrm{n}_{\mathrm{i}}<\mathrm{N} / 2<\mathrm{n}_{\mathrm{j}}$.

\section{Seedling growth studies}

Leaf extracts of $A$. philoxeroides, D. metel and $R$. dentatus, and whole plant extract of A. aspera were tested for their postemergence herbicidal potential against parthenium weed through seedling growth bioassay in pots kept in the Laboratory. Twenty seeds of parthenium weed were sown in plastic pots $(10 \mathrm{~cm}$ dia. and $10 \mathrm{~cm}$ depth) filled with $650 \mathrm{~g}$ sandy loam soil per pot initially moistened with $200 \mathrm{~mL}$ of distilled water. Each treatment was replicated four times and the experimental units were arranged in a completely randomized design. Three seedlings were maintained per pot. The $4 \mathrm{~mL}$ aqueous extract of each plant species was sprayed over 15 days-old parthenium weed seedlings. Seedlings in control treatment were also sprayed with distilled water in a similar way. Minimum and maximum temperatures during the course of experiments were $23{ }^{\circ} \mathrm{C}$ and $41{ }^{\circ} \mathrm{C}$, respectively. Ten days after spraying, 25-d-old seedlings were uprooted from pots and their shoot and root lengths were recorded. Shoot and root dry weights were recorded after drying them in an electrical oven at $65{ }^{\circ} \mathrm{C}$ for $48 \mathrm{~h}$. Germination percentage and seedling length were used to calculate seedling vigor index (SVI) by the following formula, as described by Orchard (1977):

$$
\begin{aligned}
\mathrm{SVI} & =[\text { seedling length }(\mathrm{cm}) \\
& \times \text { germination percentage }]
\end{aligned}
$$

Data Analysis: The data collected were statistically analysed using Fisher's analysis of variance technique in the MSTATC Software Program. Treatment means were compared on the basis of least significant difference (LSD) at the 0.05 probability level (Steel et al., 1997). 


\section{RESULTS AND DISCUSSION}

Germination Assay: Table 3 presents data on various germination traits, including germination percentage (GP), germination index (GI), mean germination time (MGT), and time to $50 \%$ germination $\left(\mathrm{T}_{50}\right)$. Regardless of the plant tissue used, aqueous extract of
A. aspera caused maximum reduction in parthenium GP $(92 \%)$ compared with the distilled water treated control (DWTC) (Table 3). However, A. philoxeroides delayed the germination of parthenium weed, the most $\left(\mathrm{GI}=1.6, \mathrm{MGT}=11.8 \mathrm{~d}\right.$ and $\left.\mathrm{T}_{50}=11.7 \mathrm{~d}\right)$. Extracts from leaves were most effective among the plant tissues used, regardless of

Table 3 - Effect of aqueous extracts of various plant parts on germination characteristics of Parthenium hysterophorus

\begin{tabular}{|c|c|c|c|c|c|}
\hline Plant (P) & Plant part (PP) & GP $(\%)$ & GI & MGT & $\mathrm{T} 50$ \\
\hline Control & Distilled water & $100.0 \mathrm{a}$ & $51.3 \mathrm{a}$ & $2.2 \mathrm{~m}$ & $1.6 \mathrm{i}$ \\
\hline \multirow{5}{*}{ Datura metel L. } & Root & $15.0 \mathrm{efg}$ & $3.1 \mathrm{efg}$ & $7.9 \mathrm{def}$ & $10.0 \mathrm{bc}$ \\
\hline & Stem & $10.0 \mathrm{gh}$ & $2.0 \mathrm{fg}$ & $8.0 \mathrm{def}$ & $8.5 \mathrm{~cd}$ \\
\hline & Leaf & $5.0 \mathrm{~h}$ & $0.7 \mathrm{~g}$ & $7.5 \mathrm{efg}$ & $7.0 \mathrm{def}$ \\
\hline & Fruit & $5.0 \mathrm{~h}$ & $1.6 \mathrm{fg}$ & $4.5 \mathrm{jk}$ & $4.0 \mathrm{~h}$ \\
\hline & Whole plant & $5.0 \mathrm{~h}$ & $1.1 \mathrm{~g}$ & $10.5 \mathrm{c}$ & $10.0 \mathrm{bc}$ \\
\hline \multirow{5}{*}{ Rumex dentatus L. } & Root & $21.3 \mathrm{e}$ & $4.5 \mathrm{ef}$ & $8.0 \mathrm{def}$ & $8.3 \mathrm{cde}$ \\
\hline & Stem & $10.0 \mathrm{gh}$ & $1.2 \mathrm{fg}$ & $11.9 \mathrm{~b}$ & $12.8 \mathrm{a}$ \\
\hline & Leaf & $5.0 \mathrm{~h}$ & $0.4 \mathrm{~g}$ & $14.0 \mathrm{a}$ & $13.5 \mathrm{a}$ \\
\hline & Fruit & $16.3 \mathrm{efg}$ & $3.4 \mathrm{efg}$ & 8.0 def & 6.4 efg \\
\hline & Whole plant & $12.5 \mathrm{fgh}$ & $1.8 \mathrm{fg}$ & $11.0 \mathrm{bc}$ & $10.9 \mathrm{~b}$ \\
\hline \multirow{5}{*}{ Achyranthes aspera L. } & Root & $10.0 \mathrm{gh}$ & $1.8 \mathrm{fg}$ & $9.0 \mathrm{~d}$ & $8.4 \mathrm{~cd}$ \\
\hline & Stem & $5.0 \mathrm{~h}$ & $1.1 \mathrm{~g}$ & $7.5 \mathrm{efg}$ & $7.0 \mathrm{def}$ \\
\hline & Leaf & $20.0 \mathrm{ef}$ & $5.4 \mathrm{e}$ & 6.3 ghi & $6.2 \mathrm{fg}$ \\
\hline & Fruit & $92.5 \mathrm{a}$ & $25.8 \mathrm{c}$ & 5.9 hi & $5.6 \mathrm{fgh}$ \\
\hline & Whole plant & $38.8 \mathrm{~d}$ & $12.3 \mathrm{~d}$ & $5.4 \mathrm{ij}$ & $4.8 \mathrm{gh}$ \\
\hline \multirow{4}{*}{ Alternanthera philoxeroides Gris. } & Root & $57.5 \mathrm{c}$ & $14.7 \mathrm{~d}$ & $6.8 \mathrm{fgh}$ & $7.1 \mathrm{def}$ \\
\hline & Stem & $13.8 \mathrm{efg}$ & $2.8 \mathrm{efg}$ & $8.3 \mathrm{de}$ & $7.3 \mathrm{def}$ \\
\hline & Leaf & $61.3 \mathrm{c}$ & $24.4 \mathrm{c}$ & $3.7 \mathrm{kl}$ & $2.3 \mathrm{i}$ \\
\hline & Whole plant & $81.3 \mathrm{~b}$ & $38.0 \mathrm{~b}$ & $2.8 \mathrm{~lm}$ & $1.9 \mathrm{i}$ \\
\hline \multicolumn{2}{|l|}{ LSD (PXPP) } & 7.694 & 7.69 & 2.781 & 1.171 \\
\hline \multicolumn{2}{|c|}{ Plant means } & GP $(\%)$ & GI & MGT & T50 \\
\hline \multicolumn{2}{|l|}{ Control } & $100.0 \mathrm{a}$ & $51.3 \mathrm{a}$ & $2.2 \mathrm{~d}$ & $1.6 \mathrm{e}$ \\
\hline \multicolumn{2}{|l|}{ Datura metel L. } & $8.0 \mathrm{~d}^{1 /}$ & $1.7 \mathrm{~d}$ & $7.7 \mathrm{~b}$ & $7.9 \mathrm{~b}$ \\
\hline \multicolumn{2}{|l|}{ Rumex dentatus L. } & $10.8 \mathrm{~d}$ & $1.6 \mathrm{~d}^{2 /}$ & $11.8 \mathrm{a}$ & $11.7 \mathrm{a}$ \\
\hline \multicolumn{2}{|l|}{ Achyranthes aspera L. } & $18.0 \mathrm{c}$ & $4.8 \mathrm{c}$ & $7.3 \mathrm{~b}$ & $6.6 \mathrm{c}$ \\
\hline \multicolumn{2}{|l|}{ Alternanthera philoxeroides Gris. } & $61.3 \mathrm{~b}$ & $21.1 \mathrm{~b}$ & $5.5 \mathrm{c}$ & $4.9 \mathrm{~d}$ \\
\hline \multicolumn{2}{|l|}{ LSD (Plant) } & 3.44 & 1.24 & 0.52 & 0.77 \\
\hline \multicolumn{2}{|c|}{ Plant part Means } & GP (\%) & GI & MGT & $\mathrm{T} 50$ \\
\hline \multicolumn{2}{|l|}{ Root } & $51.3 \mathrm{a}$ & $21.8 \mathrm{a}$ & $5.3 \mathrm{c}$ & $5.3 \mathrm{~b}$ \\
\hline \multicolumn{2}{|l|}{ Stem } & $37.5 \mathrm{c}$ & $14.2 \mathrm{c}$ & $7.6 \mathrm{a}$ & $7.3 \mathrm{a}$ \\
\hline \multicolumn{2}{|l|}{ Leaf } & $25.8 \mathrm{~d}$ & $11.3 \mathrm{~d}$ & $7.9 \mathrm{a}$ & $7.7 \mathrm{a}$ \\
\hline \multicolumn{2}{|l|}{ Fruit } & $38.3 \mathrm{c}$ & $16.6 \mathrm{~b}$ & $6.1 \mathrm{~b}$ & $5.5 \mathrm{~b}$ \\
\hline \multicolumn{2}{|l|}{ Whole plant } & $45.3 \mathrm{~b}$ & $16.6 \mathrm{~b}$ & $7.6 \mathrm{a}$ & $6.9 \mathrm{a}$ \\
\hline \multicolumn{2}{|l|}{ LSD (Plant part) } & 3.44 & 1.24 & 0.52 & 0.78 \\
\hline
\end{tabular}

${ }^{1 /}(92 \%) ;{ }^{2 /}(98 \%)$. In a column, values with different letters show significant difference $(p \leq 0.05)$, GP = germination percentage, $\mathrm{GI}=$ germination index, MGT $=$ mean germination time, $\mathrm{T}_{50}=$ time to $50 \%$ germination. 
species, as significantly lower GP (25.8) and GI (11.3) while higher MGT $(7.9 \mathrm{~d})$ and $\mathrm{T}_{50}$ (7.7 d) were noted with parthenium weed seeds in response to leaf extracts (Table 3). With regard to species $\mathrm{x}$ plant part interaction means, all plant parts of $R$. dentatus and root extract of A. aspera, A. philoxeroides and D. metel proved least potent inhibitors of parthenium weed germination percentage (Table 3). Germination inhibition by aqueous extracts of $A$. aspera has also been reported by Chandra et al. (2011) in broad bean (Vicia faba). Mandal \& Mondal (2011) also found that aqueous extracts from stem, root, leaves and inflorescence of $A$. philoxeroides were found to inhibit the germination of Ampelopteris prolifera.

Seedling bioassay: The significantly highest shoot length $(4.3 \mathrm{~cm})$, root length $(5.6 \mathrm{~cm})$, shoot dry weight $(18.4 \mathrm{mg})$ and root dry weight $(2.2 \mathrm{mg})$ of parthenium weeds were recorded with a distilled water sprayed control (Table 4). On the contrary, maximum inhibition in shoot length (68\%), shoot dry weight $(96 \%)$, seedling biomass $(96 \%)$, and seedling vigor index (98\%) of parthenium weeds were observed with an extract from whole plant of $A$. aspera. Whereas parthenium weed seedlings sprayed with $R$. dentatus leaf extract showed the highest suppression in root length $(70 \%)$, root dry weight $(96 \%)$ and seedling length $(68 \%)$ compared with the distilled water sprayed control. These values did not differ significantly with those noted in parthenium weed seedlings in response to A. aspera whole plant extract. Reduction in root and shoot lengths and biomass of broad bean (Vicia faba) by aqueous plant extract of A. aspera at higher concentrations was also reported by Chandra et al. (2011), whereas retardation in seedling growth of A. prolifera through the application of aqueous extracts from stem, root, leaves and inflorescence of A. philoxeroides was also found by Mandal $\&$ Mondal (2011). Seedling growth inhibition in terms of reduced plumule and radicle lengths of wheat in pea by application of aqueous extracts of root, stem and leaves of $R$. dentatus has also been documented by Umer et al. (2010). Javaid et al. (2010), in laboratory and foliar spray bioassay studies, have proved that aqueous extracts of $D$. metel have significantly reduced germination and seedling growth of parthenium weed.

Germination and seedling growth inhibitory effects of aqueous extracts of A. aspera and A. philoxeroides on parthenium weed may be due to their higher total phenolic contents (2618 to $5779 \mathrm{mgL}^{-1}$ ) (Table 1), presence of gallic (16.9 $\left.\mathrm{mgL}^{-1}\right)$,

Table 4 - Seedling growth parameters of Parthenium hysterophorus L. as influenced by postemergence application of aqueous extracts

\begin{tabular}{|c|c|c|c|c|c|c|c|c|c|}
\hline \multirow[b]{2}{*}{ Aqueous extract } & \multicolumn{9}{|c|}{ Seedling growth parameters } \\
\hline & $\begin{array}{l}\mathrm{SL} \\
(\mathrm{cm})\end{array}$ & $\begin{array}{l}\mathrm{RL} \\
(\mathrm{cm})\end{array}$ & $\begin{array}{l}\text { SFW } \\
(\mathrm{mg})\end{array}$ & $\begin{array}{l}\text { SDW } \\
(\mathrm{mg})\end{array}$ & $\begin{array}{l}\text { RFW } \\
(\mathrm{mg})\end{array}$ & $\begin{array}{l}\text { RDW } \\
(\mathrm{mg})\end{array}$ & $\begin{array}{l}\text { SDL } \\
(\mathrm{cm})\end{array}$ & $\begin{array}{l}\mathrm{SB} \\
(\mathrm{mg})\end{array}$ & SVI \\
\hline $\begin{array}{l}\text { Distilled water sprayed } \\
\text { control }\end{array}$ & $4.3 \mathrm{a}$ & $5.6 \mathrm{a}$ & $160.8 \mathrm{a}$ & $18.4 \mathrm{a}$ & $28.5 \mathrm{a}$ & $2.2 \mathrm{a}$ & 9.9 a & $20.6 \mathrm{a}$ & $986.7 \mathrm{a}$ \\
\hline $\begin{array}{l}\text { Achyranthes aspera L. whole } \\
\text { plant extract }\end{array}$ & $\begin{array}{c}1.35 \mathrm{c} \\
(68.4 \%)\end{array}$ & $\begin{array}{c}2.0 \mathrm{~d} \\
(64.3 \%)\end{array}$ & $\begin{array}{c}13.8 \mathrm{c} \\
(91.4 \%)\end{array}$ & $\begin{array}{c}0.6 \mathrm{c} \\
(96.6 \%)\end{array}$ & $\begin{array}{c}2.7 \mathrm{c} \\
(90.6 \%)\end{array}$ & $\begin{array}{c}0.2 \mathrm{c} \\
(91.8 \%)\end{array}$ & $\begin{array}{c}3.4 \mathrm{c} \\
(66.1 \%)\end{array}$ & $\begin{array}{c}0.8 \mathrm{c} \\
(96.1 \%)\end{array}$ & $\begin{array}{c}16.8 \mathrm{c} \\
(98.3 \%)\end{array}$ \\
\hline $\begin{array}{l}\text { Alternanthera philoxeroides } \\
\text { Gris. leaf extract }\end{array}$ & $\begin{array}{c}2.4 \mathrm{~b} \\
(43.3 \%)\end{array}$ & $\begin{array}{c}3.7 \mathrm{~b} \\
(33.8 \%)\end{array}$ & $\begin{array}{c}49.5 \mathrm{~b} \\
(69.2 \%)\end{array}$ & $\begin{array}{c}3.7 \mathrm{bc} \\
(79.9 \%)\end{array}$ & $\begin{array}{c}10.8 \mathrm{~b} \\
(62.3 \%)\end{array}$ & $\begin{array}{c}0.6 \mathrm{bc} \\
(72.3 \%)\end{array}$ & $\begin{array}{c}6.1 \mathrm{~b} \\
(37.9 \%)\end{array}$ & $\begin{array}{c}4.3 \mathrm{bc} \\
(79.1 \%)\end{array}$ & $\begin{array}{l}30.6 \mathrm{bc} \\
(96.9 \%)\end{array}$ \\
\hline Datura metel L. leaf extract & $\begin{array}{c}4.00 \mathrm{a} \\
(6.8 \%) \\
\end{array}$ & $\begin{array}{c}2.8 \mathrm{c} \\
(49.5 \%) \\
\end{array}$ & $\begin{array}{c}58.4 \mathrm{~b} \\
(63.7 \%) \\
\end{array}$ & $\begin{array}{c}6.4 \mathrm{~b} \\
(65.5 \%) \\
\end{array}$ & $\begin{array}{c}11.1 \mathrm{~b} \\
(61.2 \%)\end{array}$ & $\begin{array}{c}0.6 \mathrm{~b} \\
(72.7 \%) \\
\end{array}$ & $\begin{array}{c}6.8 \mathrm{~b} \\
(30.9 \%) \\
\end{array}$ & $\begin{array}{c}7.00 \mathrm{~b} \\
(66.3 \%) \\
\end{array}$ & $\begin{array}{c}34.1 \mathrm{~b} \\
(96.5 \%) \\
\end{array}$ \\
\hline $\begin{array}{l}\text { Rumex dentatus L. leaf } \\
\text { extract }\end{array}$ & $\begin{array}{c}1.4 \mathrm{c} \\
(66.5 \%)\end{array}$ & $\begin{array}{c}1.7 \mathrm{~d} \\
(70.5 \%)\end{array}$ & $\begin{array}{c}19.1 \mathrm{c} \\
(88.1 \%)\end{array}$ & $\begin{array}{c}1.3 \mathrm{c} \\
(92.8 \%)\end{array}$ & $\begin{array}{c}0.8 \mathrm{c} \\
(97.1 \%)\end{array}$ & $\begin{array}{c}0.1 \mathrm{~b} \\
(96.4 \%)\end{array}$ & $\begin{array}{c}3.1 \mathrm{c} \\
(68.8 \%)\end{array}$ & $\begin{array}{c}1.4 \mathrm{c} \\
(93.2 \%)\end{array}$ & $\begin{array}{c}43.5 \mathrm{~b} \\
(95.6 \%)\end{array}$ \\
\hline $\mathrm{LSD}_{0.05}$ & 0.308 & 0.654 & 25.08 & 3.565 & 5.104 & 0.5202 & 0.769 & 4.005 & 14.00 \\
\hline
\end{tabular}

In a column, values with different letters show significant difference $(p \leq 0.05)$ by the LSD Test, Figures in parenthesis show $\%$ reduction over control in the respective column, $\mathrm{SL}=$ shoot length, $\mathrm{RL}=$ root length, $\mathrm{SFW}=$ shoot fresh weight, $\mathrm{SDW}=$ shoot dry weight, $\mathrm{RFW}=$ root fresh weight, $\mathrm{RDW}=$ root dry weight, $\mathrm{SDL}=$ seedling length, $\mathrm{SB}=$ seedling biomass, $\mathrm{SVI}=$ seedling vigor index. 
caffeic (7.4 $\left.\mathrm{mg} \mathrm{L}^{-1}\right)$, chromatotropic (13.8 to $\left.63.8 \mathrm{mg} \mathrm{L}^{-1}\right), p$-coumaric $\left(10.5 \mathrm{mg} \mathrm{L}^{-1}\right)$, $m$-coumaric $\left(3.1 \mathrm{mg} \mathrm{L}^{-1}\right)$, syringic $\left(9.2 \mathrm{mg} \mathrm{L}^{-1}\right)$ and 4-hydroxy-3-methoxy benzoic (24.8 to $118.6 \mathrm{mg} \mathrm{L}^{-1}$ ) acids in their aqueous extracts (Table 2). Achyranthine is the major alkaloid present in all tissues of $A$. aspera whereas other allelochemicals in various plant parts are phenolics, oleonolic acid, dihydroxy ketones, long chain compounds and saponin A and B (Ali, 1993; Srivastav et al., 2011).

Based on preemergence and postemergence activities against parthenium, whole plant extract of $A$. aspera and leaf extract A. philoxeroides are concluded as herbicidal. Therefore, these extracts should be further tested alone or in combination with existing synthetic herbicides for sustainable and eco-friendly management of parthenium weeds.

\section{ACKNOWLEDGMENTS}

This manuscript is part of a thesis by the principal author for his $\mathrm{PhD}$ degree in the Department of Agronomy, University of Agriculture, Faisalabad, Pakistan. The authors of this manuscript greatly acknowledge Dr. Razia Riaz, Research Officer, Hi Tech. Laboratory, University of Agriculture, Faisalabad, Pakistan for her kind cooperation in providing facilities for HPLC studies.

\section{LITERATURE CITED}

ALI, M. Chemical investigation of Achyranthes aspera Linn. Oriental J. Chem., v. 9, n. 1, p. 84-85, 1993.

ANJUM, T. et al. Biological control of Parthenium I. Effect of Imperata cylindrica on distribution, germination and seedling growth of Parthenium hysterophorus L. Intern. J. Agric. Biol., v. 7, n. 3, p. 448-450, 2005.

CHANDRA, S. et al. Hormetic impact of Achyranthes aspera L. extracts on seed and seedling traits of Vicia faba L.

Columban J. Life Sci., v. 2, n. 1/2, p. 87-91, 2011.

CHIPPENDALE, J. F.; PANETTA, F. D. The cost of parthenium weed to the Queensland cattle industry. Plant Protec. Quart., v. 9, n. 2, p. 73-76, 1994.

EVANS, H. C. Parthenium hysterophorus: a review of its weed status and the possibilities for biological control.

Biocont News Infor., v. 18, n. 3, p. 89-98, 1997.
FAROOQ, M. et al. Thermal hardening: a new seed vigor enhancement tool in rice. J. Integr. Plant Biol., v. 47, n. 2, p. 187-193, 2005.

HUSSAIN, F.; GADOON, M. A. Allelopathic effects of Sorghum vulgare. Pers. Oecologia, v. 51, n. 3, p. 284-288, 1981.

KHAN, H. et al. Chemical control of Parthenium hysterophorus L. at different growth stages in non-cropped area. Pakistan J. Bot., v. 44, n. 5, p. 1721-1726, 2012.

KHAN, H. et al. Socio-economic impacts of Parthenium weed (Parthenium hysterophorus L.) in Peshawar valley, KPK.

Pakistan J. Weed Sci. Res., v. 19, n. 3, p. 275-293, 2013.

KHAN, H. et al. Distribution of Parthenium weed in Peshawar valley, KPK-Pakistan. Pakistan J. Bot., v. 46, n. 1, p. 81-90, 2014.

JAVAID, A.; ANJUM, T. Parthenium hysterophorus L. - a noxious alien weed. Pakistan. J. Weed Sci. Res., v. 11, n. 3/ 4, p. 81-87, 2005.

JAVAID, A. et al. Biological control of Parthenium II. Allelo6pathic effect of Desmostachya bipinnata on distribution and early seedling growth of Parthenium hysterophorus L. Intern. J. Biol. Biotechnol., v. 2, n. 2, p. 459-463, 2005.

JAVAID, A. et al. Causes of rapid spread of Parthenium hysterophorus L. in Pakistan and possible control measures a review. Pakistan J. Bot., v. 39, n. 7, p. 2611-2618, 2007.

JAVAID, A. et al. Herbicidal effects of extracts and residue incorporation of Datura metel against parthenium weed. Nat. Prod. Res., v. 24, n. 15, p. 1426-1437, 2010.

KATHIRESAN, R. Ecology and control of Parthenium hysterophorus invasion in veeranum command area. Indian $\mathbf{J}$. Weed Sci., v. 40, n. 1-2, p. 78-80, 2008.

MANDAL, A.; MONDAL, A.K. Taxonomy and ecology of obnoxious weed Alternanthera Philoxeroides Grisebach (Family Amaranthaceae) on spore germination in Ampelopteris Prolifera (Ketz.) Cop. Adv. Biores., v. 2, n. 1, p. 103-110, 2011.

MASUM, S. M. et al. Threats of Parthenium hysterophorus on agro-ecosystems and its management: a review. Intern. J. Agric. Crop Sci., v. 6, n. 11, p. 684-697, 2013.

DEZFULI, P. M. et al. Influence of priming techniques on seed germination behavior of maize inbred lines (Zea mays L.). ARPN J. Agric. Biol. Sci., v. 3, n. 3, p. 22-25, 2008.

NIGATU, L. et al. Impact of Parthenium hysterophorus on grazing land communities in north-eastern Ethiopia. Weed Biol. Manage., v. 10, n. 3, p. 143-152, 2010.

Planta Daninha, Viçosa-MG, v. 34, n. 1, p. 109-116, 2016 
ORCHARD, T. Estimating the parameters of plant seedling emergence. Seed Sci. Technol., v. 5, n. 1, p. 61-69, 1977.

QUAZI, S. M.; KHAN, M. A. Suppression of Parthenium hysterophorus L. by Alternanthera polygonoides (L) R. Br. Bioinfolet, v. 7, n. 1, p. 91, 2010.

RANDHIR, R.; SHETTY, K. Developmental stimulation of total phenolics and related antioxidant activity in light and dark germinated maize by natural elicitors. Proc. Biochem., v. 40, n. 5, p. 1721-1732, 2005.

RIAZ, T.; JAVAID A. Prevalence of invasive parthenium weed in district Hafizabad, Pakistan. J. An. Plant Sci., v. 20, n. 2, p. 90-93, 2010.

SAFDAR, M. E. et al. Tree species as a potential source of bio-herbicides for controlling Parthenium hysterophorus L.

Nat. Prod. Res., v. 27, n. 22, p. 2154-2156, 2013.

SCOTT, S. J. et al. Review of data analysis methods for seed germination. Crop Sci., v. 24, n. 6, p. 1192-1198, 1984.

SHABBIR, A.; BAJWA, R. Parthenium invasion in Pakistan a threat still unrecognized. Pakistan J. Bot., v. 39, n. 7 , p 2519-2526, 2007.

SHAFIQUE, S. et al. Tagetes erectus L. - a potential resolution for management of Parthenium hysterophorus $\mathrm{L}$.

Pakistan J. Bot., v. 43, n. 2, p. 885-894, 2011.

SHARMA, R. Performance of different herbicides for control of congress grass (Parthenium hysterophorus L.) in noncropped areas. Indian J. Weed Sci., v. 35, n. 3-4, p. 242-245, 2003.
SINHA, N. K.; SINGH, S. Allelopathic effects of Xanthium strumarium on Parthenium hysterophorus. Indian J. Plant Physiol., v. 9, n. 3, p. 313-315, 2004.

SRIVASTAV, S. et al. Achyranthes aspera-An important medicinal plant: A review. J. Nat. Prod. Plant Res., v. 1, n. 1, p. 1-14, 2011.

STEEL, R. G. D. et al. Principles and procedures of statistics. A Biometrical Approach. 3.ed. New York: McGraw Hill Book, 1997. 672 p.

SURESH, P. et al. Bronchial provocation with Parthenium pollen extract in bronchial asthma. Indian J. Chest. Dis. Allied Sci., v. 36, p. 104, 1994.

SWAIN, D. et al. Effects of Physalis minima on Parthenium hysterophorus. Allelop. J., v. 15, n. 2, p. 275-283, 2005.

TAMADO, T. et al. Interference by the weed Parthenium hysterophorus L. with grain sorghum: Influence of weed density and duration of competition. Intern. J. Pest Manage., v. 48, n. 1, p. 183-188, 2002.

UMER, A. et al. Evaluation of allelopathic potential of some selected medicinal species. African J. Biotechnol., v. 9, n. 37, p. 6194-6206, 2010.

VIVEK R. S. et al. Effect of weed interference on weeds and productivity of blackgram (Phaseolus mungo). Indian J. Weed Sci., v. 40, n. 1-2, p. 65-67, 2008.

XUAN, T. D. et al. Biological control of weeds and plants pathogens in paddy rice by exploiting plant allelopathy: an overview. Crop Protec., v. 24, n. 3, p. 197-206, 2005. 